\title{
The Balance of Spiritual and Nutritional Needs Improves the Well-being of Cancer Patients during Chemotherapy
}

\author{
Aat Sriati ${ }^{1}$, Laili Rahayuwati ${ }^{1,3}$, Maria Komariah ${ }^{1,2}$, Hendrawati $^{1}$, Iceu Amira ${ }^{1}$, Sukma Senjaya ${ }^{1}$, Indra Maulana ${ }^{1 *}$, Efri Widianti ${ }^{1}$ \\ ${ }^{1}$ Department of Mental Health Nursing, Faculty of Nursing, Universitas Padjadjaran, Sumedang, Indonesia; ${ }^{2}$ Department \\ of Fundamental Nursing, Faculty of Nursing, Universitas Padjadjaran, Sumedang, Indonesia; ${ }^{3}$ Departement of Community \\ Nursing, Faculty of Nursing, Universitas Padjadjaran, Sumedang, Indonesia
}



\section{Introduction}

The health service which is an integral part of Indonesia's health care system must facilitate the interactive process between patients, community needs, and the provider demands. Therefore, it is necessary to create an ideal, holistic, and integrative health care system to establish patient empowerment.

However, it is undoubted that Indonesian health care, which mostly deals with disease prevalence and mortality, is in jeopardy, particularly when it comes to malignant tumors or cancers. As a result, breast cancer, which is the most common cancer among women has acquired a rise in incidence, specifically in Indonesia, Japan, Malaysia, Philippines, Singapore, Sri Lanka, and Taiwan. It was reported by WHO in 2015 that, on the subject of death and illness ratio, 508.000 women died of breast cancer in 2011.

Furthermore, breast cancer generally occurs in the group of women who have gotten to their menopausal age, although recently, it has been found in many young women (less than 25 years old), while only a few cases occurred in men. Moreover, it also affects the well-being and prosperity of a woman in terms of physique, emotion, psychology, social, and spiritual aspects, particularly in women of working age, playing the roles of a wife or mother. Although breast cancer is known as an illness mostly occurring in developing or developed countries, around $50 \%$ of breast cancer patients and $58 \%$ of death caused by breast cancer happens in developing countries [1], [2].

Subsequently, this research begins with a qualitative observation in understanding experiences and identifying the need for therapy in breast cancer patients. Specifically, the aims of this research are as follows: (1) providing information on bio-medics-based health service to breast cancer patients; (2) identifying factors pertinent to breast cancer patients during and after chemotherapy; (3) offering a standpoint to health professionals on breast cancer patients and their needs for an improvement in palliative care quality; (4) developing useful information to design and apply the palliative services for breast cancer patients. 


\section{Methods}

The design of this research is qualitative with a case study approach aiming at analyzing the needs of breast cancer patients during chemotherapy. Each sample consists of outpatients or inpatients as well as those who have completed therapy and reside in a neighborhood.

This sample tends to be non-probable, purposefully about 19 patients who have experience in breast-cancer-related therapy. However, two patients, unfortunately, died along the journey (complete information was thus gathered from the remaining 17 patients), but some crucial comments were obtained from them as well. Hence, the number of subjects recruited in this research hinged on the process, where the exact number was considered sufficient only when saturation point was reached.

Furthermore, qualitative data was collected through in-depth interviews as well as an observation of the patient's condition. The result was on a contentanalysis-oriented basis, where the following steps were carried out: (1) observing symbols and utterances of the subjects; (2) constructing the data which gave meaning to the symbol or utterance both verbally or nonverbally regarding the subject's needs; (3) describing and verifying conclusions; (4) re-validating the interview results; (5) making reports based on the existing research criteria. Importantly, in qualitative research, the process of data collection and analysis occurs at the same time, as an interpretation was conducted to describe the research.

This research has been ethically approved by the Ethical Commission of Health Research in Faculty of Medicine Universitas Padjadjaran, No. 277/UN6. C1.3.2/KEPK/PN/2015. The ethical decision regarding be sought information was suitable with the aim of the research, and the subjects involved, who were breast cancer patients and health professionals. The preparation of informed consent to complete ethical considerations was conducted to provide proper information. Additionally, the participants had the freedom to choose whether to take part in the research or not.

\section{Results}

The result showed only a few patients were able to explain the therapy process. Generally, they simply followed what they were advised to do by family members or hospital staff. Their understanding of cancer and its cure was exceptionally narrow, with little information being obtained from television means, having been deprived of the chance to know the origins of cancer in detail. Other pieces of information regarding cancer were also obtained from their allegedly believable sources such as husbands, spouses, friends, or neighbors.

Additionally, sources of information during treatment process was obtained through individual inquiry by the patients' family. This included television, media, or internet surfing as well as story exchanges between patients who experience chemotherapy. Some patients also enquired about their illnesses and the treatment from doctors, but only a small proportion was satisfied with the responses given. In addition, information was obtained as well from nurses on the types of medications and their usage.

Furthermore, some of the patients claimed that they had experienced several treatment steps such as chemotherapy, surgery, and radiation. They also admitted that the chemotherapy was done to destroy the cancer cells, while surgery and radiation were done to dispose and dry the cancer cells out.

Moreso, It was discovered that nearly all breast cancer patients have the "fear" of breast cancer surgery. Some of them claimed it was better not to embark on the surgical process, knowing that breasts are one of women's physical images or icons. Therefore, taking them away would mean losing their dignity or physical value.

Similarly, another difficult aspect was experiencing chemotherapy, considering its unbearable impact of side effects. The patients claimed that the effects of chemotherapy were not only nausea and regurgitation but also hair loss and baldness, alongside drastic weight loss and murky changes in skin color.

Subsequently, most of the patients claimed having the worst physical experience during chemotherapy. During a direct interview, a patient said: "I feel nauseous, unable to eat, drink. My body dropped the first time I did my chemo. When the next schedule is coming, I can't stand it anymore. The idea of "dropping" and being treated in the hospital previously scares the nerves out of me. I have to eat nutrition-rife food before doing the next chemo". Another patient further stated: "To me, experiencing chemotherapy is the hardest of all (other than radiation or surgery). I have many complaints every time I do it: dizziness, sprue, even eye infection. Besides, the effects of chemotherapy are tougher than those of radiation or surgery. I can't even taste anything while eating".

Although, in viewing the effects of chemotherapy, each patient had varying responses, but in viewing the treatment process, almost all of them stated positively how they could see a spark of hope in recovering.

Additionally, there are several drugs used by breast cancer patients during chemotherapy, these drugs include: a) tamoxifen (prevents estrogen), a medication typically used for hormonal therapy, 
consumed in the forms of tablets or syrup. It also serves in blocking estrogen from binding with cancer cells. This drug could also cause some similar side effects such as dizziness, nausea, regurgitation, a sensation of heat, sweats, and hot flushes. Sometimes, it retains specific side effect such as a drastic shift in menstrual cycle; b) dexamethasone (repression of pain, according to some patients) which is a group of corticosteroid drugs, that serves to prevent the release of inner-body substances that causes inflammation, particularly in cancer patients; c) ranitidine (a curative drug for ulcer) which is used to repress symptoms and illnesses associated with excessive gastric acid. Such excesses might subject the walls of the digestive tract to irritation and inflammation, which will further lead to illnesses such as peptic ulcers, duodenum ulcers, general ulcers, heartburn, and other digestive problems. This medicine works by repressing the amount of excessive gastric acid to thin out the pain and enable the wound in the gastric wall to heal slowly. Besides, ranitidine could also be used to prevent symptoms of digestive problems caused by certain food intake; d) ondansetron, a drug used to prevent and cure nausea and regurgitation caused by chemotherapy, radiotherapy, or surgical side effects. In addition, nausea and regurgitation are caused by bodily natural compounds called 5-hydroxytryptamine (5HT3) or serotonin, whose amount increases when experiencing therapy or surgery. It will also react to the $5 \mathrm{HT} 3$ receptor which lies on the small intestine or brain, causing mild nausea. Therefore, this drug will block the reaction of serotonin to receptors, blocking nausea and vomiting from occurring.

In this regard, the minority of respondents stated that since they experienced therapy, they depend on the given prescriptions only. They were also very strict to themselves in terms of avoiding consumption of other medicines, as one of them puts it: "since chemotherapy, I dare not try other kinds of drug. If it comes to stomachache, I just bear it as it will otherwise clash with the chemo drugs".

\section{The Balance of Spiritual and Nutritional Needs in Improving the Well-Being of Cancer Patients}

The condition of the illness being more severe daily makes the informant realize that death probably will pick up but efforts were made to keep living. As revealed by the following informant: ".... I do not know when I die, the important thing I have tried is to run the Shari'a. The essence is only Allah who has, Allah, is omniscient". This same situation was also experienced by other informants in this research. Additionally, a series of conventional treatment procedures beginning with chemotherapy, mastectomy, and radiation therapy affected the informants' lives. Various complaints, such as lymphedema, jaundice, anemia, and associated discomfort, were expressed by those who had completed radiation therapy. Due to this, an informant attempted to reinforce his spiritual foundation by instructing his children to study beside him until he fell asleep. As revealed by this informant: ".... if I died, I can still listen to my son's voice studying Qur'an. I am so calm “.

Moreover, the improvement of spiritual health and psychological prosperity is another attempt constantly embarked by the patients. Eating patterns or diet as well as other nutrition fulfilling efforts were also included. This is relevant to what Ahmad, et.al (2011); Witdiawati, Rahayuwati, Pritasari, (2016) stated about most respondents in their research believing that obesity $(52,7 \%)$, less physical activity (48.7\%), and high-fat diet (63.2\%) were related to cancer restoration. Another attempt was in minimizing the side effects of breast cancer, such as pain.

Furthermore, this result showed that not many patients are willing to try to fulfill their nutrition needs effectively and sufficiently, as some specific result to "fighting" the dwindling appetite. On the other side, most patients stated the loss of their appetites made it difficult for them to fulfill their required nutritional intake. Concerning the observation of 2 non-survivors, it was shown that their body condition was feeble, mostly due to an inappropriate amount of nutrition intake. The patient's family supplied the nutrition needed by the patient by preparing a variety of foods, but the quality and quantity were regarded insufficient. Based on this observation, it was also clear that the patients were usually malnourished, which has an impact on their long-term survival. Meanwhile, some survivors showed a better physical condition, as two of them after the 6th therapy did not show much physical deficiency in terms of skin and nail color and of flexibility in carrying out their routines. These patients noted that, in addition to chemotherapy, good nutrition which includes eating organic eggs three times a day, fruit and vegetables three or four times a day, and increasing the consumption of nutrition-rich food should be prioritized throughout the recovery process. Subsequently, a disciplined diet could also be considered as an alternative to maintain and heal bodily conditions as shown by several survivors within the age of $43-76$, who were physically active. This set of survivors arranged a daily menu consisting of varying amounts of carbohydrates, fruits, vegetables (specifically juice), to help those who lose appetite digest better. Among these survivors, one claimed, after a 10-year of the post-therapy period the following as was said: "every day, I make myself prepare main consumption like 6 kinds of carbohydrate during therapy: rice, porridge, corn, cassava, tuber, sago; two spoons each. However, not all of them can be digested perfectly. Some are thrown out, but, no matter how much you puke, if the amount of carbohydrate is abundant, the rest of it will remain inside". 
The minority of patients were able to survive in dealing with the effects of chemotherapy by choosing to create a strict diet plan. Besides, the observation showed that a good nutritional status will affect the patient's physical strength throughout the therapy.

\section{Discussion}

The objectives of the therapy are as follows: a) preventing cancer cells from self-replicating and spreading; b) disrupting rapidly-growing cell replication; c) having maximum efficacy with as minimum side effects as possible. Therefore, there are two types of chemotherapy: first, which is applied only after surgery to destroy cancer cells, and second, before surgery to scrutinize tumors. Breast cancer therapy is all about anti-cancer drugs delivered through infusion to outpatients. Also, doctors might consider prescribing tablets to be taken home.

Furthermore, the function of therapy and its side effects must be taken into consideration by patients. The patients showed be informed that side effects will occur only when the drugs destroy cancer cells as well as rapidly-replicating normal cells (such cancer cells), particularly in hair, mouth and intestinal mucous, and blood cells. The effects are, however, temporary, usually normalized from a week in the post-therapy period. Generally, some commonly experienced effects are related to: a) digestive system: nausea, regurgitation, stomatitis, diarrhea, constipation; b) myelosuppression: anemia, leukopenia, thrombocytopenia; c) fatigue: neuropathy, myopathy; d) hair loss.

Moreso, Cordova, and Andrykowsky, conducted extensive research investigating the traumatic consequences of cancer and discovered that the diagnosis and treatment of cancer can be an extremely stressful experience for most individuals. They found that $49 \%$ of their sample of women with breast cancer who were 6 to 60 months post-treatment indicated that they experienced repeated disturbing memories of cancer treatment and the experiences they had during that period [3]. Additionally, seven percent reported having repeated disturbing dreams of cancer treatment or their experience with fear of recurrence, death, surgery, and adjuvant side effects of the treatment. In addition, 35\% indicated that they experienced physical reactions such as nausea when something reminded them of cancer treatment or their experiences. It was also discovered that higher scores were associated with lower social support, larger precancerous trauma history, less time since breast cancer treatment completion, and more advanced disease.

Subsequently, from some literature, it was reported that spirituality is a strong predictor and promoter of psychological health [4]; [5]. It is in line with the results from[6],[7], [8]which states when the patient reaches the end of life, there becomes a need to fulfill spiritual needs. In addition to this, previous research had suggested that spirituality can increase the patient's endurance to mental health crisis after cancer diagnosis and treatment [9]; [10];[11]. Further, Islamic Psychological Management refers to the religious rituals commonly practiced by Muslims to manage the psychological impact of the disease. This is a method of relaxation that includes Islamic principles such as prayer, recitation of the Qur'an, and the meditation of dhikr, to obtain a calm state and awareness response. Thus, this intervention will be given to breast cancer patients experiencing chemotherapy to reduce the psychological impact, specifically anxiety and mental well-being.

Moreover, the improvement of spiritual health and psychological prosperity is another attempt constantly carried out by the patients. Likewise, eating patterns or diet as well as other nutrition fulfilling efforts were also included. These improvements are relevant to what [12]; [7] stated that most respondents believe that obesity $(52,7 \%)$, less physical activity $(48.7 \%)$, and a high-fat diet (63.2\%) are related to cancer restoration. Another attempt is minimizing the effect of side effects associated with breast cancer, such as pain.

In addition, the research result demonstrates that only a few breast cancer patients during their chemotherapy understood the importance of nutrition needs. Besides, some of these patients consume food or go through diet, based simply on prescription with the belief that they could eat anything. Therefore, this problem should be highlighted; as some kinds of food are proper to be consumed only if chosen personally by the patient or family member who serves the menu during treatment and recovery. Despite this, few of them can describe how their diet affects their health. Some survivors even agree that with the right quantity of nutrition, they might live a better life. Subsequently, given the deterioration of the immune system and the increased risk of infection in patients, it is necessary to prepare ahead of time. Hence, the improvement of the patient's nutritional condition is considered one of the most significant ways to enhance their immune system. Therefore, a balanced intake and requirements for biological functions should be maintained. Otherwise, it will have an impact on their metabolism, as well as their physical and mental health.

In addition, to improve the nutritional status, it is necessary to have specific nutritional therapy, which serves in mending the immune system after a breakdown as cancer patients need enough energy from a high amount of carbohydrate and protein. Besides, the sufficient amount of protein in an immunelacking patient is crucial for preventing and countering infection from occurring.

Moreso, it is expected that by maintaining good nutritional status, the life expectancy of cancer patients 
will increase. Subsequently, in reference to some other research results, cancer patients who experience weight loss of more than $5 \%$ have a shorter life expectancy than those who do not, and such loss affects theActivity of Daily Life status and their life quality.

Furthermore, it was shown by some research that malnutrition and cachexia (the risk of high malnutrition to cancer patients) often occur in cancer patients $(24 \%$ at an early stage and $>80 \%$ at a chronic stage) [13].In addition, the cause of malnutrition in cancer patients are generally divided into 2 categories: 1) dwindling of food intake and malabsorption; 2) problems in the metabolism process [14].

Additionally, Nurse servicing, commonly known as nursing health service with nutritionists, must focus on a measured eating pattern, decreasing the number of patients' complaints and fixing the general condition. The objective of diet in breast cancer is to optimize nutrient status by eating the proper amount of food with sufficient nutrients following the patients' receptivity, as well as preventing weight loss, nausea, regurgitation, and diarrhea.

In this regard, the recommendation for food containing antioxidants is necessary for breast cancer patients, for the substances that destroy cancer cells, protect, and mend body cells due to free radicals, are commonly found in many kinds of vitamins and minerals. In addition, several experts stated that cell damage only causes chronic illnesses such as cancer, transportation problem, or arthritis. Likewise, free radicals might also disrupt the immune system, hence, vitamins such as $A, B, C, D, E$, and $K$, are important to restore that system [15].

\section{Conclusion}

It is recommended for further research to conduct an investigation about the multifactorial impacts on the degradation of life quality and even mortality in breast cancer patients. The discovery based on observation showed that spiritual supports and nutritional status play an influential factor in the success of medical therapy that includes radiation and chemotherapy.

As a result, it is expected that spiritual therapies and adequate nutritional intake for cancer patients would begin early, even before they experience chemotherapy. Another important aspect is that the healthcare personnel should be able to give basic information as well as answers to queries regarding various types of spiritual treatment and nutritional therapy.

\section{References}

1. Zaidi Z, Dib HA. The worldwide female breast cancer incidence and survival, 2018. AACR; 2019. http://doi. org/10.1158/1538-7445

2. DeSantis CE, Bray F, Ferlay J, Lortet-Tieulent J, Anderson BO, Jemal A. International variation in female breast cancer incidence and mortality rates. Cancer Epidemiol Prev Biomark. 2015;24(10):1495-506. http://doi.org/10.1158/1055-9965. EPI-15-0535

PMid:26359465

3. Cordova MJ, Andrykowski MA. Responses to cancer diagnosis and treatment: posttraumatic stress and posttraumatic growth. Semin Clin Neuropsychiatry. 2003;8(4):286-96.

PMid:14613054

4. Figueroa LR, Davis B, Baker S, Bunch JB. The influence of spirituality on health care-seeking behaviors among African Americans. ABNF J. 2006;17(2):82.

PMid:18402349

5. Sankhe A, Dalal K, Save D, Sarve P. Evaluation of the effect of Spiritual care on patients with generalized anxiety and depression: Arandomized controlled study. Psychol Health Med. 2017;22(10):118691. https://doi.org/10.1080/13548506.2017.1290260

6. Tsitsis N, Lavdaniti M. Quality of life in women with breast cancer. Int J Caring Sci. 2014;7(1):135.

7. Witdiawati W, Rahayuwati L, Sari SP. Studi kualitatif pola kehidupan pasien kanker payudara. J Keperawatan Padjadjaran. 2017;5(1):73-85. https://doi.org/10.24198/jkp.v5i1.352

8. Rahayuwati L, Ibrahim K, Nurhidayah I, Hendrawati S. The correlation of socio demographic and knowledge factors toward therapy options among breast cancer patients. J Keperawatan Padjadjaran. 2020;8(3):1582. https://doi.org/10.24198/jkp.v8i3.1582

9. Pedersen $C G$, Christensen $S$, Jensen $A B$, Zachariae R. In god and CAM we trust. Religious faith and use of complementary and alternative medicine (CAM) in a nationwide cohort of women treated for early breast cancer. J Relig Health. 2013;52(3):9911013. https://doi.org/10.1007/s10943-012-9569-x PMid:22318498

10. Meraviglia M. Effects of spirituality in breast cancer survivors. Oncol Nurs Forum. 2006;33(1):E1-7. https://doi.org/10.1188/06. onf.e1-e7

PMid: 16470229

11. Movafagh A, Heidari MH, Abdoljabbari M, Mansouri N, Taghavi A Karamatinia A, et al. Spiritual therapy in coping with cancer as a complementary medical preventive practice. J Cancer Prev. 2017;22(2):82.

PMid:28698861

12. Ahmad F, Muhammad MB, Abdullah AA. Religion and spirituality in coping with advanced breast cancer: perspectives from Malaysian Muslim women. J Relig Health. 2011;50(1):36-45. https://doi.org/10.1007/s10943-010-9401-4

13. Miniguano-TrujilloA, Salazar F, Torres R, Arias P, Sotomayor K. An integer programming model to assign patients based on mental health impact for tele-psychotherapy intervention during the Covid-19 emergency. Health Care Manag Sci. 2021;24(2):286304. https://doi.org/10.1007/s10729-020-09543-z PMid:33839993

14. Shike M. Nutrition therapy for the cancer patient. Hematol Clin. 1996;10(1):221-34. PMid:8821569

15. Arends J, Bachmann $P$, Baracos $V$, Barthelemy $N$, Bertz $H$, Bozzetti $F$, et al. ESPEN guidelines on nutrition in cancer patients. Clin Nutr. 2017;36(1):11-48. 